\title{
Quantum implications of a scale invariant regularization
}

\author{
D. M. Ghilencea* \\ Theoretical Physics Department, National Institute of Physics and Nuclear Engineering Bucharest, \\ Măgurele 077125, Romania
}

(Received 24 January 2018; published 12 April 2018)

\begin{abstract}
We study scale invariance at the quantum level in a perturbative approach. For a scale-invariant classical theory, the scalar potential is computed at a three-loop level while keeping manifest this symmetry. Spontaneous scale symmetry breaking is transmitted at a quantum level to the visible sector (of $\phi$ ) by the associated Goldstone mode (dilaton $\sigma$ ), which enables a scale-invariant regularization and whose vacuum expectation value $\langle\sigma\rangle$ generates the subtraction scale $(\mu)$. While the hidden $(\sigma)$ and visible sector $(\phi)$ are classically decoupled in $d=4$ due to an enhanced Poincaré symmetry, they interact through (a series of) evanescent couplings $\propto \epsilon$, dictated by the scale invariance of the action in $d=4-2 \epsilon$. At the quantum level, these couplings generate new corrections to the potential, as scale-invariant nonpolynomial effective operators $\phi^{2 n+4} / \sigma^{2 n}$. These are comparable in size to "standard" loop corrections and are important for values of $\phi$ close to $\langle\sigma\rangle$. For $n=1,2$, the beta functions of their coefficient are computed at three loops. In the IR limit, dilaton fluctuations decouple, the effective operators are suppressed by large $\langle\sigma\rangle$, and the effective potential becomes that of a renormalizable theory with explicit scale symmetry breaking by the DR scheme (of $\mu=$ constant).
\end{abstract}

DOI: 10.1103/PhysRevD.97.075015

\section{INTRODUCTION}

It is a common view that the Standard Model (SM) is only a low-energy effective theory and "new physics" could arise at some scale below $M_{\text {Planck. The scale of "new }}$ physics" can be the vacuum expectation value (vev) of a scalar field $\sigma$ present beyond the SM spectrum. It is then natural to ask how the Higgs mass is protected from large quantum corrections associated with $\langle\sigma\rangle .^{1}$ One long-held answer is $\mathrm{TeV}$ supersymmetry. ${ }^{2}$

Scale invariance may also protect the Higgs mass against large quantum corrections. This starts from the observation that for a vanishing Higgs mass parameter, SM has an increased symmetry: it is scale invariant. This means that the classical action is invariant under a transformation: $x \rightarrow$ $\rho^{-1} x, \phi \rightarrow \rho^{d_{\phi}} \phi\left(d_{\phi}\right.$ is the mass dimension of $\left.\phi\right)$. Scale symmetry was noticed to play a role in protecting the electroweak scale [3-6] with classically scale-invariant extensions of the SM considered in [7-26]. But to address

\footnotetext{
*dumitru.ghilencea@cern.ch

${ }^{1}$ In the absence of "new physics" below $M_{\text {Planck }}$ and ignoring gravity, SM has no hierarchy problem.

${ }^{2} \mathrm{TeV}$-SUSY models have large fine-tuning [1], which cannot coexist with a good data fit $\chi^{2} /$ dof $\approx 1[2]$.

Published by the American Physical Society under the terms of the Creative Commons Attribution 4.0 International license. Further distribution of this work must maintain attribution to the author(s) and the published article's title, journal citation, and DOI. Funded by SCOAP.
}

the mass hierarchy problem, one must go beyond the classical scale symmetry, since the counterterms are actually dictated by the quantum symmetry. This could naturally protect [27] the Higgs mass from large quantum corrections [28] associated with a high scale $\langle\sigma\rangle$ of "new physics". For studies of quantum scale invariance (broken spontaneously) and applications to SM, see [29-38].

Our goal is to further study models in which the classical scale symmetry is extended at the quantum level and is broken spontaneously. ${ }^{3}$ In such a theory, all scales are generated by the fields' vev's. Such a theory can predict ratios of scales (vev's) only, in terms of ratios of dimensionless couplings. A hierarchy of physical mass scales can then be generated by a hierarchy of such couplings. The latter is easier to protect by a symmetry (e.g., an enhanced Poincaré symmetry [39]) and is more fundamental than a hierarchy of (dimensionful) physical scales. Indeed, in a fundamental theory, any physical scale should ultimately be determined in terms of dimensionless couplings and fields vev's.

Since scale symmetry is broken in the real world, we assume it is broken spontaneously. A flat direction exists, and the spectrum contains the associated Goldstone boson

\footnotetext{
${ }^{3}$ Classical scale symmetry is often broken by quantum calculations since the UV regulator breaks it explicitly. The classical flat direction is then lifted, and a light pseudo-Goldstone boson exists. See, for example, [5]. We do not follow this approach and implement instead a quantum scale symmetry.
} 
(dilaton, hereafter $\sigma$ ) beyond the spectrum of the initial model. The subtraction scale $\mu$ (used in loop calculations) that would break quantum scale symmetry explicitly, is replaced by the field $\sigma$ which thus maintains the scale symmetry at a quantum level and after spontaneous breaking generates $\mu \sim\langle\sigma\rangle$, see Englert et al. [29]. This gives a scale-invariant regularization (SR). ${ }^{4}$

In this paper, we discuss further consequences of the original idea of Englert et al. [29]. The SR scheme can be applied to any gauge theory, although we restrict our study to a scalar theory. We study more quantum effects in this scheme and stress the role of symmetries. In $d=4$, the hidden sector (of the dilaton $\sigma$ ) is classically decoupled from the visible sector (of Higgs-like $\phi$ ) by invoking an enhanced Poincaré symmetry $P_{v} \times P_{h}$ of these two sectors [39]. At the quantum level, the manifest scale symmetry of the action in $d=4-2 \epsilon$ introduces evanescent couplings $\propto \epsilon \tilde{\sigma} /\langle\sigma\rangle$ of the hidden to the visible $\operatorname{sector}^{5}$ ( $\tilde{\sigma}$ : dilaton fluctuations). The SR scheme is thus reformulated into an ordinary DR scheme of $\mu=$ constant $(\alpha\langle\sigma\rangle)$ plus an additional field $(\sigma)$ with an infinite series of evanescent couplings to the visible sector.

At the quantum level, such evanescent couplings have physical effects. When these couplings multiply poles of momentum integrals, they generate new (finite or infinite) counterterms, all scale invariant. For example, one finds nonpolynomial operators generated radiatively, such as $\phi^{2 n+4} / \sigma^{2 n}, n \geq 1$ (but also higher derivative operators suppressed by $\sigma$ ). They can transmit scale symmetry breaking to the visible sector. Such operators can be understood via their Taylor expansion about $\sigma=\langle\sigma\rangle+\tilde{\sigma}$, when they become polynomial. Scale symmetry acts at the quantum level as an organizing principle that resums the polynomial ones. We shall study closer these operators, since they are important at large $\phi$. Because of their presence, the quantum scale invariant theory is nonrenormalizable.

We compute in a manifest scale invariant way, the quantum corrections to the scalar potential in a two-loop order (diagrammatically) and three-loop (via CallanSymanzik equation) for a scale-invariant classical theory. The two-loop (three-loop) potential contains effective operators as finite (infinite) counterterms, respectively. In the infrared (IR) decoupling limit of the dilaton (large $\langle\sigma\rangle$ ), effective operators vanish; one then recovers the effective potential and trace anomaly of a renormalizable theory (if classical theory was so) with only classical scale invariance and explicit scale symmetry breaking (SSB) by DR with $\mu=$ constant. The combined role of quantum scale invariance and enhanced Poincaré symmetry in protecting the scalar mass at large $\langle\sigma\rangle$ is also reviewed.

\footnotetext{
${ }^{4}$ Versions of this scheme were used in [30-37] (in some cases, classical Higgs-dilaton mixing was present which concealed the enhanced Poincaré symmetry and the effects discussed below).

${ }^{5}$ By evanescent coupling, we understand a coupling that is nonzero in $d=4-2 \epsilon$ and is vanishing in $d=4$.
}

Since $M_{\text {Planck }}$ breaks scale symmetry, this analysis is valid for field values well below this scale. One should extend this study to a Brans-Dicke-Jordan theory of gravity with nonminimal coupling where the dilaton vev $\langle\sigma\rangle$ spontaneously fixes $M_{\text {Planck }}$. We restrict the analysis to a perturbative (quantum) scale symmetry. At very high momentum scales, some couplings (e.g., hypercharge) may become nonperturbative, but such a scale is above $M_{\text {Planck }}$, where flat spacetime description used here fails anyway.

\section{FROM CLASSICAL TO QUANTUM SCALE INVARIANCE}

\section{A. Implementing quantum scale invariance}

Consider a classical scale invariant action, e.g., a toy model or the SM with vanishing Higgs mass parameter, extended by the dilaton $\sigma$. We assume that there is no classical interaction between the visible sector (of fields $\phi_{j}$ ) and the hidden sector (of dilaton $\sigma$ ). Then

$$
S=\int d^{4} x L_{v}\left(\phi_{j}, \partial \phi_{j}\right)+\int d^{4} y L_{h}(\sigma, \partial \sigma)
$$

The action in $d=4$ has an enhanced Poincaré symmetry $\left(P_{v} \times P_{h}\right)$ associated with both sectors, which forbids a classical coupling $\lambda_{m} \phi_{j}^{2} \sigma^{2}$. Such a coupling can be naturally set to $\lambda_{m}=0$ and remains so at the quantum level. ${ }^{6}$ "protected" by this symmetry [39].

Below we work with the canonical dilaton $\sigma$ related to the actual Goldstone by $\sigma=\langle\sigma\rangle e^{\tau}$, so that it transforms in a "standard" way under scaling while $\tau$ transforms with a shift

$$
x \rightarrow \rho^{-1} x, \quad \sigma \rightarrow \rho \sigma, \quad \tau \rightarrow \tau+\ln \rho .
$$

The most general potential for $\sigma$ allowed by scale invariance in $d=4$ is then $\kappa_{0} e^{4 \tau} \sim \lambda_{\sigma} \sigma^{4}$. But Poincaré symmetry in the dilaton sector demands a flat potential, so $\lambda_{\sigma}=0$ [40]. Demanding spontaneous scale symmetry breaking $\langle\sigma\rangle \neq 0$ means "we live" along a flat direction. Ultimately this is a tuning of the cosmological constant and is present anyway in, e.g., $\mathrm{TeV}$ supersymmetry. The details of how $\sigma$ acquires a vev are not relevant below.

At the quantum level, it is natural to use the dilaton to generate dynamically the subtraction scale $\propto\langle\sigma\rangle$ in order to preserve scale symmetry during quantum calculations [29]. We use DR in $d=4-2 \epsilon$, then the only possibility dictated by dimensional arguments ${ }^{7}$ is

$$
\mu=z \sigma^{2 /(d-2)},
$$

\footnotetext{
${ }^{6}$ Technically, $\beta_{\lambda_{m}} \propto \lambda_{m}$ at two loop [36].

${ }^{7} \mu$ has a mass dimension one, while $\sigma$ and $\langle\sigma\rangle$ have a dimension $(d-2) / 2$.
} 
with $z$ is an arbitrary dimensionless parameter (scaling factor); it keeps track of the vev of $\sigma$ after SSB. The $d=4$ potential $V\left(\phi_{j}\right)$ of the visible sector is then analytically continued to $d=4-2 \epsilon$, into $\mu^{2 \epsilon} V\left(\phi_{j}\right)$. Therefore, the potential in $d=4-2 \epsilon$ is actually

$$
\tilde{V}\left(\phi_{j}, \sigma\right)=\left[z \sigma^{2 /(d-2)}\right]^{4-d} V\left(\phi_{j}\right)
$$

and becomes a function of $\sigma$.. This ensures the $d=4$ couplings remain dimensionless in $d=4-2 \epsilon$ and can be used for perturbative calculations. Therefore, the visible $\left(\phi_{j}\right)$ and hidden $(\sigma)$ sectors have evanescent couplings dictated by the scale symmetry alone of the (regularized) action in $d=4-2 \epsilon$. To see these couplings, expand (4) in powers of $\epsilon$ (loops) and then in terms of fluctuations $\tilde{\sigma}$ about the vev $\langle\sigma\rangle$ of $\sigma$,

$$
\begin{aligned}
\tilde{V}\left(\phi_{j}, \sigma\right)= & \mu_{0}^{2 \epsilon}\left[1+2 \epsilon\left(\eta-\frac{1}{2} \eta^{2}+\frac{1}{3} \eta^{3}+\mathcal{O}\left(\eta^{4}\right)\right)\right. \\
& \left.+\epsilon^{2}\left(2 \eta+\eta^{2}-\frac{4}{3} \eta^{3}+\mathcal{O}\left(\eta^{4}\right)\right)+\mathcal{O}\left(\epsilon^{3}\right)\right] V\left(\phi_{j}\right)
\end{aligned}
$$

where

$$
\mu_{0}=z\langle\sigma\rangle^{\frac{1}{1-\epsilon}}, \quad \sigma=\langle\sigma\rangle+\tilde{\sigma}, \quad \eta=\frac{\tilde{\sigma}}{\langle\sigma\rangle} .
$$

A scale invariant regularization is then reexpressed as an ordinary DR scheme with $\mu=\mu_{0}$ plus an extra field $(\sigma)$ with (infinitely many) evanescent couplings Eq. (5). Since the lhs is scale invariant, so is the rhs if one does not truncate the expansion in field fluctuations. In practice, one can still use a truncated expansion (see below). From Eq. (5), one can read the new vertices of evanescent interactions $\propto \epsilon^{n}(n \geq 1)$, between $\tilde{\sigma}$ and $\phi_{j}$ and the Feynman rules of the scale invariant quantum theory. ${ }^{8}$ While these interactions vanish in $d=4$ or in the dilaton decoupling limit $(\eta \rightarrow 0)$, at the loop level have physical effects.

At the quantum level, a coupling proportional to $\epsilon^{n}$, $(n \geq 1)$ in an amplitude can bring new corrections to it when multiplying the poles $1 / \epsilon^{k}$ of the integrals over loop momenta. One generates finite quantum corrections (if $n=k)$ or new poles/counterterms $(n<k)$ beyond those of the theory with $\mu=$ constant. If $n=k$, a scattering amplitude that involves the dilaton depends only on the couplings of the initial $d=4$ theory, without new parameters needed (counterterm couplings). This can be used to set strong lower bounds on the scale $\langle\sigma\rangle$.

Since the new couplings are suppressed, $\eta \sim 1 /\langle\sigma\rangle$, the counterterms are higher dimensional. They must however respect the scale symmetry of the lhs of Eq. (5); one can

\footnotetext{
${ }^{8}$ Field-dependent masses and propagators also acquire $\epsilon$ shifts, relevant at loop level.
}

then "restore" this symmetry "broken" by working with the truncation of the rhs expression, by simply replacing $1 /\langle\sigma\rangle \rightarrow 1 / \sigma$ in their expression. Therefore, the new counterterms of the theory are suppressed by powers of $\sigma$ and are nonpolynomial in fields; log terms in $\sigma$ are also possible, however (see later).

For example, for $V(\phi)=\lambda \phi^{4} / 4$ !, a first counterterm is found by inserting a single internal line of $\tilde{\sigma}$ in an amplitude, which brings a factor $(\epsilon /\langle\sigma\rangle)^{2}$; if this multiplies a $1 / \epsilon^{3}$ pole from a three-loop momentum integral, it generates a $1 / \epsilon$ pole and a corresponding counterterm $\phi^{6} / \sigma^{2}$ for the six-point amplitude $\left(\phi^{6}\right)$ [37]. By the same argument, finite quantum corrections appear at two loops (if due to dynamics of $\sigma$ ) or even one loop (due to scale symmetry alone).

Since the theory is scale invariant and so it has no dimensionful couplings, diagrams that would otherwise be proportional to masses automatically vanish. Then the only possibility to construct scale invariant $d=4$ counterterms that are suppressed by powers of $\sigma$ is to involve appropriate powers $\phi^{n}, n>4$ and higher derivatives of $\phi$ and $\tilde{\sigma}$. Therefore, the new counterterms are found on dimensional grounds as

$$
\sum_{n, m \geq 0} a_{m n} \frac{\partial^{2 n} \alpha^{m+4}}{\sigma^{2 n+m}}, \quad \alpha=\phi, \sigma
$$

where the derivatives act in all possible ways in the numerator. This includes the dilaton-dilaton scattering $\left(\partial_{\mu} \sigma\right)^{4} / \sigma^{4}$ (see $a$-theorem [41]) which emerges at three loops.

We see that quantum scale-invariant theories are nonrenormalizable [37], unlike their counterpart with $\mu=$ constant which is not quantum scale invariant but is renormalizable (if initial $d=4$ action was so). The latter case is recovered in the limit of a large $\langle\sigma\rangle$, when fluctuations $\tilde{\sigma}$ decouple, see Eq. (5). This picture also applies to gauge theories.

\section{B. One-loop potential}

Let us first review the quantum corrections to the potential in a scale invariant toy model at one loop, before going to higher loops. Consider $L$ below in $d=4$ for a scalar $\phi$

$$
L=\frac{1}{2}\left(\partial_{\mu} \phi\right)^{2}+\frac{1}{2}\left(\partial_{\mu} \sigma\right)^{2}-V(\phi), \quad V(\phi)=\frac{\lambda}{4 !} \phi^{4}
$$

In $d=4-2 \epsilon$, the potential becomes $\tilde{V}$ of Eq. (4) with $V$ above, so $\phi$ and $\sigma$ do interact as dictated by the scale symmetry of analytically continued $L$. The one-loop potential is then 


$$
\begin{aligned}
V_{1} & =\tilde{V}-\frac{i}{2} \int \frac{d^{d} p}{(2 \pi)^{d}} \operatorname{Tr} \ln \left[p^{2}-\tilde{V}_{\alpha \beta}+i \varepsilon\right] \\
& =\tilde{V}+\frac{1}{4 \kappa} \sum_{s=\phi, \sigma} \tilde{M}_{s}^{4}\left(\frac{-1}{\epsilon}+\ln \frac{\tilde{M}_{s}^{2}}{c_{0}}\right), \\
\kappa & =(4 \pi)^{2}
\end{aligned}
$$

where $c_{0}=4 \pi e^{3 / 2-\gamma_{E}} . \tilde{M}_{S}^{2}$ are field-dependent (masses) ${ }^{2}$, eigenvalues of the second derivatives matrix $\tilde{V}_{\alpha \beta}$, with $\alpha, \beta=\phi, \sigma$. One eigenvalue $\tilde{M}_{\sigma}^{4} \propto \epsilon^{2}$; thus, it cannot generate counterterms at one loop. Then

$$
V_{1}=\tilde{V}+\mu^{2 \epsilon} \frac{V_{\phi \phi}^{2}}{4 \kappa}\left\{-\frac{1}{\epsilon}+\left(\overline{\ln } \frac{V_{\phi \phi}}{(z \sigma)^{2}}-\frac{1}{2}\right)\right\},
$$

with $\quad V_{\phi \phi}=\frac{1}{2} \lambda \phi^{2}$,

with $\overline{\ln } A \equiv \ln A /\left(4 \pi e^{1-\gamma_{E}}\right)$. It is important to note that the factor $\mu^{2 \epsilon}$ is a function of $\sigma$ [see Eq. (3)] and maintains scale invariance $^{9}$ in $d=4-2 \epsilon$. Here, we work in the minimal subtraction scheme (MS). Thus, the (scale-invariant) counterterm is

$$
\delta L_{1}=-\mu^{2 \epsilon} \frac{1}{4 !} \delta_{\lambda}^{(1)} \lambda \phi^{4} \quad \text { with } \delta_{\lambda}^{(1)} \equiv Z_{\lambda}-1=\frac{3 \lambda}{2 \kappa \epsilon} .
$$

Then the one-loop potential in $d=4$ is

$$
U=V(\phi)+\frac{1}{4 \kappa} V_{\phi \phi}^{2}\left[\overline{\ln } \frac{V_{\phi \phi}}{(z \sigma)^{2}}-\frac{1}{2}\right]
$$

and is indeed scale invariant. Since dimensionless $z$ keeps track of the presence of $\langle\sigma\rangle$, the one-loop beta function $\beta_{\lambda}^{(1)}$ is found by demanding the bare coupling $\lambda^{B}=$ $\mu(\sigma)^{2 \epsilon} \lambda Z_{\lambda} Z_{\phi}^{-2}$ be independent of scaling parameter $z$,

$$
\frac{d \lambda^{B}}{d \ln z}=0 \Rightarrow \beta_{\lambda}^{(1)}=\frac{d \lambda}{d \ln z}=\frac{3}{\kappa} \lambda^{2},
$$

which is identical to the result for the case $\mu=$ constant. $^{10}$ The Callan-Symanzik (CS) equation for a scale-invariant theory [33] is easily verified,

$$
\frac{d U}{d \ln z}=\left(\frac{\partial}{\partial \ln z}+\beta_{\lambda}^{(1)} \frac{\partial}{\partial \lambda}\right) U=O\left(\lambda^{3}\right) .
$$

Consider now the limit when the dilaton decouples. For this, Taylor expand the potential for $\sigma=\langle\sigma\rangle+\tilde{\sigma}$ where $\tilde{\sigma}$ are field fluctuations. The result is

\footnotetext{
${ }^{9}$ This can also be relevant if one wanted to define and use instead a nonminimal subtraction scheme.

${ }^{10}$ Unlike in theories with no dilaton (with explicit SSB by quantum corrections), $\beta_{\lambda}=0$ is not a necessary condition for having scale symmetry in our case here $[32,33]$ since the spectrum is extended to include a dilaton (spontaneous SSB); thus, a nonzero $\beta_{\lambda}$ does not mean the theory cannot be scale invariant.
}

$$
U=V(\phi)+\frac{1}{4 \kappa} V_{\phi \phi}^{2}\left[\overline{\ln } \frac{V_{\phi \phi}}{(z\langle\sigma\rangle)^{2}}-\frac{1}{2}\right]+\Delta U
$$

with

$$
\Delta U=\frac{1}{4 \kappa} V_{\phi \phi}^{2}\left(-\frac{\tilde{\sigma}}{\langle\sigma\rangle}+\frac{1}{2} \frac{\tilde{\sigma}^{2}}{\langle\sigma\rangle^{2}}+\cdots\right) .
$$

For $\tilde{\sigma} \ll\langle\sigma\rangle, \Delta U=0$, and we recover the ColemanWeinberg result of a $d=4$ renormalizable theory obtained in the DR scheme with $\mu=$ constant $(=z\langle\sigma\rangle)$, with explicit SSB. Obviously, the CS equation is still respected. One can then proceed to impose boundary conditions, to define the quartic self-coupling at $\phi=\langle\sigma\rangle: \lambda_{\langle\sigma\rangle}=\partial^{4} U /\left.\partial \phi^{4}\right|_{\phi=\langle\sigma\rangle}$, as usual.

The analysis is similar if more fields $\phi_{j}$ are present, of potential $V\left(\phi_{j}\right)$. The result is found from Eqs. (15) and (16) by replacing $V_{\phi \phi}$ by the eigenvalues of matrix $V_{i j}=$ $\partial^{2} V / \partial \phi_{i} \partial \phi_{j}$ and summing over them. Again the dilaton does not contribute directly at one loop, but enforces the scale invariance of $U$. The second term in the CS equation in (14) is now a sum over all quartic couplings in $V$. Including fermions and gauge bosons is immediate by extending the sum over field dependent masses, with appropriate factors.

\section{Two-loop potential}

The two-loop correction to the potential of $\phi$ can be written as

$$
V_{2}=V_{2}^{a}+V_{2}^{b}+V_{2}^{c}
$$

with the diagrams below computed from the background field method ${ }^{11}$

$$
V_{2}^{a}=\frac{i}{12} \circlearrowright \quad V_{2}^{b}=\frac{i}{8} \bigcirc ; \quad V_{2}^{c}=\frac{i}{2} \circlearrowright
$$

The vertices and propagators in these diagrams receive evanescent corrections from the dilaton field, as seen from the background field expansion. We Taylor expand

$$
\begin{aligned}
\tilde{V}(\phi+\delta \phi, \sigma+\delta \sigma)= & \tilde{V}(\phi, \sigma)+\tilde{V}_{\alpha} s_{\alpha}+\frac{1}{2} \tilde{V}_{\alpha \beta} s_{\alpha} s_{\beta} \\
& +\frac{1}{3 !} \tilde{V}_{\alpha \beta \gamma} s_{\alpha} s_{\beta} s_{\gamma}+\frac{1}{4 !} \tilde{V}_{\alpha \beta \gamma \rho} s_{\alpha} s_{\beta} s_{\gamma} s_{\rho} \\
& +\cdots
\end{aligned}
$$

where $s_{\alpha}=\delta \phi, \delta \sigma$ are the actual field fluctuations. The vertices $\tilde{V}_{\alpha \beta \ldots}=\partial \tilde{V} / \partial \alpha \partial \beta \ldots(\alpha, \beta, \ldots=\phi, \sigma)$ contain

\footnotetext{
${ }^{11}$ We use the approach of [36] but without a classical coupling $\lambda_{m} \phi^{2} \sigma^{2}$.
} 
terms proportional to powers of $\epsilon$, e.g., $\tilde{V}_{\phi \phi \sigma}=$ $\lambda\left(\phi^{2} / \sigma\right) \epsilon+\mathcal{O}\left(\epsilon^{2}\right)$. The propagators, obtained from the inverse of the matrix $\left(p^{2} \delta_{\alpha \beta}-\tilde{V}_{\alpha \beta}\right)$, also acquire $\epsilon$-dependent shifts. We retain all these corrections up to and including $\mathcal{O}\left(\epsilon^{2}\right)$; these can multiply the poles of the loop integrals $\left(1 / \epsilon^{2}\right.$ or $\left.1 / \epsilon\right)$ to generate finite quantum corrections, ${ }^{12}$ as discussed in Sec. II A. Here, we shall identify these corrections. One finds

$$
V_{2}=\mu^{2 \epsilon} \frac{\lambda^{3} \phi^{4}}{32 \kappa^{2}}\left\{-\frac{3}{\epsilon^{2}}+\frac{2}{\epsilon}+\mathcal{O}\left(\epsilon^{0}\right)\right\}
$$

with $\mu^{2 \epsilon}$ a function of $\sigma$ which ensures the scale invariance in $d=4-2 \epsilon$, see Eq. (3). The counterterm is scale invariant, and in the MS scheme is given by

$$
\delta L_{2}=\frac{1}{2}\left(\partial_{\mu} \phi\right)^{2} \delta_{\phi}^{(2)}-\mu^{2 \epsilon} \frac{1}{4 !} \lambda \phi^{4} \delta_{\lambda}^{(2)}
$$

and

$$
\delta_{\lambda}^{(2)}=\frac{\lambda^{2}}{\kappa^{2}}\left(\frac{9}{4 \epsilon^{2}}-\frac{3}{2 \epsilon}\right), \quad \delta_{\phi}^{(2)}=\frac{-\lambda^{2}}{24 \kappa^{2} \epsilon} .
$$

From these and with the coefficients $Z_{\lambda}=1+\delta_{\lambda}, Z_{\phi}=$ $1+\delta_{\phi}$ and since $\lambda^{B}=\mu^{2 \epsilon} \lambda Z_{\lambda} Z_{\phi}^{-2}, d \lambda^{B} / d(\ln z)=0$, one obtains the two-loop corrected beta function

$$
\beta_{\lambda}=\frac{3}{\kappa} \lambda^{2}-\frac{17}{3 \kappa^{2}} \lambda^{3} .
$$

$\beta_{\lambda}$ is identical to that of the $\phi^{4}$ theory with $\mu=$ constant (no dilaton) [42-44]. No new poles (i.e., counterterms) are generated at two loop beyond those of the theory with $\mu=$ constant.

The two-loop potential we find is

$$
\begin{aligned}
U= & \frac{\lambda}{4 !} \phi^{4}\left\{1+\frac{3 \lambda}{2 \kappa}\left(\overline{\ln } \frac{V_{\phi \phi}}{(z \sigma)^{2}}-\frac{1}{2}\right)\right. \\
& +\frac{3 \lambda^{2}}{4 \kappa^{2}}\left(4+A_{0}-4 \overline{\ln } \frac{V_{\phi \phi}}{(z \sigma)^{2}}+3 \overline{\ln }^{2} \frac{V_{\phi \phi}}{(z \sigma)^{2}}\right) \\
& \left.+\frac{5 \lambda^{2}}{\kappa^{2}} \frac{\phi^{2}}{\sigma^{2}}+\frac{7 \lambda^{2}}{24 \kappa^{2}} \frac{\phi^{4}}{\sigma^{4}}\right\},
\end{aligned}
$$

where ${ }^{13} A_{0}=-(8 / 3) \sqrt{3} \mathrm{Cl}_{2}(\pi / 3) \approx-4.688 \cdots$.

Equation (24) is an interesting result. First, $U$ is scale invariant. The last two terms in $U$ are new, finite two-loop corrections in the form of nonpolynomial operators $\left(\phi^{6} / \sigma^{2}, \phi^{8} / \sigma^{4}, \ldots\right)$ and cannot be removed by a different subtraction scheme. These terms are independent of the dimensionless subtraction parameter $z$ and bring

\footnotetext{
${ }^{12} \mathrm{New} 1 / \epsilon$ poles from $(\epsilon-$ shifts $) \times 1 / \epsilon^{2}$ do not emerge here, unless a classical mixing $\phi-\sigma$ exists.

${ }^{13}$ The Clausen function $\mathrm{Cl}_{2}$ is defined as $\mathrm{Cl}_{2}[x]=$ $-\int_{0}^{x} d \theta \ln |2 \sin \theta / 2|$.
}

corrections beyond those obtained for $\mu=$ constant (of explicit SSB). Their presence is easily understood in the light of the discussion in Sec. II A. The field-dependent masses entering the loop calculation, as eigenvalues of the second derivative matrix $\tilde{V}_{\alpha \beta}$, contain terms suppressed by $\mu^{2} \sim \sigma^{2}$, since the sole dependence on $\sigma$ is $\tilde{V} \sim \sigma^{\epsilon}$. This explains the presence of positive powers of $\sigma$ only in the denominators of the nonpolynomial terms. Even the simplest quantum scale invariant theory is then nonrenormalizable (unlike the case with $\mu=$ constant which is renormalizable but not quantum scale invariant).

The one-loop terms which are $O(\lambda / \kappa)$ (for $\log \sim 1$ ) dominate the new two-loop nonpolynomial terms if

$$
\frac{\lambda}{\kappa} \frac{\phi^{n}}{\sigma^{n}}<1, \quad n=2,4
$$

The nonpolynomial terms can be larger than the "standard" two-loop correction; they are comparable in size for $\phi \sim \sigma$. Higher loops are expected to generate more such operators of larger powers and with new couplings (if they are counterterms ${ }^{14}$ ). They are relevant if one is interested in the stability of the potential at large field values $\phi \sim\langle\sigma\rangle$. The nonpolynomial terms vanish in the limit $\phi \ll \sigma$.

The result in Eq. (24) can be Taylor expanded about the vev of $\sigma$ using $\sigma=\langle\sigma\rangle+\tilde{\sigma}$. Retaining only the leading term corresponds to decoupling the dilaton. Then

$$
\begin{aligned}
U= & \frac{\lambda}{4 !} \phi^{4}\left\{1+\frac{3 \lambda}{2 \kappa}\left(\overline{\ln } \frac{V_{\phi \phi}}{\langle z \sigma\rangle^{2}}-\frac{1}{2}\right)\right. \\
& \left.+\frac{3 \lambda^{2}}{4 \kappa^{2}}\left(4+A_{0}-4 \overline{\ln } \frac{V_{\phi \phi}}{\langle z \sigma\rangle^{2}}+3 \overline{\ln }^{2} \frac{V_{\phi \phi}}{\langle z \sigma\rangle^{2}}\right)\right\} \\
& +\mathcal{O}\left(\frac{1}{\langle\sigma\rangle}\right) .
\end{aligned}
$$

Ignoring $\mathcal{O}(1 /\langle\sigma\rangle)$ terms, Eq. (26) is the "standard" twoloop result obtained for $\mu=$ constant (no dilaton, explicit SSB) in the MS scheme [45], more exactly for $\mu=z\langle\sigma\rangle$. The difference between Eqs. (24) and (26) is made of higher dimensional operators suppressed by large $\langle\sigma\rangle$; these suppressed terms are responsible for maintaining manifest scale invariance of (24).

The generic form of the Callan-Symanzik equation is [33]

$$
\left(\frac{\partial}{\partial \ln z}+\beta_{\lambda_{j}} \frac{\partial}{\partial \lambda_{j}}+\phi \gamma_{\phi} \frac{\partial}{\partial \phi}+\sigma \gamma_{\sigma} \frac{\partial}{\partial \sigma}\right) U\left(\phi_{j}, \sigma, \lambda_{j}, z\right)=0,
$$

and we use it to check the result of (24). Here ${ }^{15}$

\footnotetext{
${ }^{14}$ This is discussed in the next section.

${ }^{15}$ At two loop, $\gamma_{\phi}$ is $\gamma_{\phi}^{(2)}=-\lambda^{2} /\left(12 \kappa^{2}\right)$, from Eq. (22).
} 


$$
\gamma_{\phi}=\frac{d \ln \phi}{d \ln z}=\frac{-1}{2} \frac{d \ln Z_{\phi}}{d \ln z}
$$

To check Eq. (27), first use Eq. (24) to introduce a decomposition $U=V+V^{(1)}+V^{(2)}+V^{(2, n)}$ to denote the tree level $(V)$, one loop $\left(V^{(1)}\right)$, the "usual" two-loop correction with $\mu \rightarrow z \sigma\left(V^{(2)}\right)$, and finally, the new finite two-loop correction $\left(V^{(2, n)}\right)$ of the nonpolynomial operators [the sum of the last two terms in (24)]. Equation (27) is decomposed into three equations,

$$
\begin{aligned}
& \frac{\partial V^{(1)}}{\partial \ln z}+\beta_{\lambda}^{(1)} \frac{\partial V}{\partial \lambda}=O\left(\lambda^{3}\right) \\
& \frac{\partial V^{(2)}}{\partial \ln z}+\left(\beta_{\lambda}^{(2)} \frac{\partial}{\partial \lambda}+\gamma_{\phi}^{(2)} \phi \frac{\partial}{\partial \phi}+\gamma_{\sigma}^{(2)} \sigma \frac{\partial}{\partial \sigma}\right) V+\beta_{\lambda}^{(1)} \frac{\partial V^{(1)}}{\partial \lambda} \\
& \quad=O\left(\lambda^{4}\right) \\
& \frac{\partial V^{(2, \mathrm{n})}}{\partial \ln z}=O\left(\lambda^{4}\right),
\end{aligned}
$$

where $\beta_{\lambda}^{(k)}, k=1,2,$. denote the $k$-loop correction to the beta function of $\lambda$ (similar for $\gamma_{\phi, \sigma}^{(2)}$ ). We verified that Eqs. (29)-(31) are respected. This is a consistency check of Eq. (24).

The Callan-Symanzik equation is also respected in the non-scale-invariant case, Eq. (26), where $\mu=$ constant $(\mu=z\langle\sigma\rangle$, explicit SSB). This is obvious from the above check because $z$ is tracking exactly this scale, and the nonpolynomial terms in (24) are $z$ independent. ${ }^{16}$

\section{Three-loop potential}

In this section, we use the three-loop Callan-Symanzik equation for the scalar potential to identify the three-loop correction to the potential without doing the diagrammatic calculation. As in the two-loop case, this correction is a sum of two terms $V^{(3)}+V^{(3, n)} \cdot V^{(3)}$ is the "usual" three-loop correction obtained with $\mu=$ constant $[45,46]$, but with the formal replacement $\mu \rightarrow z \sigma ; V^{(3, n)}$ is a new correction that contains nonpolynomial terms. To find these, we use the three-loop counterterms for this theory nicely computed in [37]

$$
\begin{aligned}
\delta L_{3}= & \frac{1}{2} \delta_{\phi}^{(3)}\left(\partial_{\mu} \phi\right)^{2} \\
& -\mu^{2 \epsilon}\left(\frac{1}{4 !} \delta_{\lambda}^{(3)} \lambda \phi^{4}+\frac{1}{6} \delta_{\lambda_{6}}^{(3)} \lambda_{6} \frac{\phi^{6}}{\sigma^{2}}+\frac{1}{8} \delta_{\lambda_{8}}^{(3)} \lambda_{8} \frac{\phi^{8}}{\sigma^{4}}\right) .
\end{aligned}
$$

$\delta L_{3}$ is scale invariant in $d=4-2 \epsilon$ (as it should) because $\mu$ depends on $\sigma$, Eq. (3). The terms $\phi^{6} / \sigma^{2}$ and $\phi^{8} / \sigma^{4}$ are expected since they were present as finite operators at two loop; also,

\footnotetext{
${ }^{16}$ This changes at three loops, see $V^{(3, n)}$ in the next section.
}

$$
\delta_{\phi}^{(3)}=-\frac{\lambda^{3}}{4 \kappa^{3}}\left(\frac{1}{6 \epsilon^{2}}-\frac{1}{12 \epsilon}\right)
$$

in the MS scheme, giving $\gamma_{\phi}^{(3)}=\lambda^{3} /\left(16 \kappa^{3}\right)$ and

$$
\delta_{\lambda_{6}}^{(3)}=\frac{3}{2} \frac{\lambda^{4}}{\lambda_{6} \kappa^{3} \epsilon}, \quad \delta_{\lambda_{8}}^{(3)}=\frac{275}{864} \frac{\lambda^{4}}{\lambda_{8} \kappa^{3} \epsilon} .
$$

With $\lambda_{6}^{B}=\mu^{2 \epsilon}(\sigma) \lambda_{6} Z_{\lambda_{6}} Z_{\phi}^{-3} Z_{\sigma}$, etc., and with $(d / d \ln z) \lambda_{6}^{B}=0$, we find

$$
\begin{aligned}
& \beta_{\lambda_{6}}=\frac{\lambda^{2} \lambda_{6}}{2 \kappa^{2}}+\frac{\lambda^{3}}{\kappa^{3}}\left(9 \lambda-\frac{3}{8} \lambda_{6}\right) \\
& \beta_{\lambda_{8}}=\frac{2 \lambda^{2} \lambda_{8}}{3 \kappa^{2}}+\frac{\lambda^{3}}{4 \kappa^{3}}\left(\frac{275}{36} \lambda-2 \lambda_{8}\right) .
\end{aligned}
$$

Both beta functions have a two-loop part (hereafter denoted $\left.\beta_{\lambda_{6,8}}^{(2, n)} \sim 1 / \kappa^{2}\right)$ that is absent if $\lambda_{6,8}=0$ in the classical Lagrangian, which is our case here ${ }^{17}$; then the three-loop part (hereafter $\beta_{\lambda_{6,8}}^{(3, n)} \sim 1 / \kappa^{3}$ ) is induced by $\lambda$ alone. These beta functions enter in the CS equations in the presence of $\lambda_{6}$ and $\lambda_{8}$, due to their associated counterterms. In their presence, Eq. (29) is unaffected, but Eq. (30) is modified such as $V$ is now replaced by

$$
V \rightarrow V+\Delta V, \quad \Delta V=\frac{\lambda_{6}}{6} \frac{\phi^{6}}{\sigma^{2}}+\frac{\lambda_{8}}{8} \frac{\phi^{8}}{\sigma^{4}},
$$

and $\beta_{\lambda_{6,8}}^{(2, n)}$ are also included in the first term under the big bracket of (30). Using these and the "new" $V$ above, one immediately sees that (30) is verified for nonzero $\lambda_{6,8}$.

Further, there is a CS equation at order $\lambda^{4}$ for $\left(V^{(3)}+V^{(3, n)}\right)$, which we divide into two CS equations, Eqs. (37) and (40) below. One equation is for the "usual" correction $V^{(3)}$ and is identical to that obtained for $\mu=$ constant $(=z\langle\sigma\rangle)$

$$
\begin{aligned}
& \frac{\partial V^{(3)}}{\partial \ln z}+\beta_{\lambda}^{(1)} \frac{\partial V^{(2)}}{\partial \lambda}+\beta_{\lambda}^{(2)} \frac{\partial V^{(1)}}{\partial \lambda}+\beta_{\lambda}^{(3)} \frac{\partial V}{\partial \lambda}+\gamma_{\phi}^{(2)} \frac{\partial V^{(1)}}{\partial \ln \phi} \\
& +\gamma_{\phi}^{(3)} \frac{\partial V}{\partial \ln \phi}=\mathcal{O}\left(\lambda_{j}^{5}\right) .
\end{aligned}
$$

We integrate (37) to find $V^{(3)}$ up to an unknown "constant" of integration term $\propto \mathcal{Q}$

$$
\begin{aligned}
V^{(3)}= & \frac{\lambda^{4} \phi^{4}}{\kappa^{3}}\left\{\mathcal{Q}+\left(\frac{97}{128}+\frac{9}{64} A_{0}+\frac{\zeta[3]}{4}\right) \overline{\ln } \frac{V_{\phi \phi}}{(z \sigma)^{2}}\right. \\
& \left.-\frac{31}{96} \overline{\ln }^{2} \frac{V_{\phi \phi}}{(z \sigma)^{2}}+\frac{9}{64} \overline{\ln }^{3} \frac{V_{\phi \phi}}{(z \sigma)^{2}}\right\} .
\end{aligned}
$$

\footnotetext{
${ }^{17}$ Otherwise, the terms $\phi^{6} / \sigma^{2}$ and $\phi^{8} / \sigma^{4}$ would have been counterterms already at two loop, in Eq. (24).
} 
$\mathcal{Q}$ can be read from the "usual" three-loop computation at $\mu=$ constant [45] in the MS scheme,

$$
\begin{aligned}
\mathcal{Q} \equiv & \frac{1}{288}\left\{\frac{-1673}{8}+\frac{9}{4} A_{0}\left(A_{0}-4\right)+\frac{34 \pi^{4}}{15}+8 \pi^{2} \ln ^{2} 2\right. \\
& \left.-8 \ln ^{4} 2-192 \operatorname{Li}_{4}\left[\frac{1}{2}\right]+72 \zeta[3]\right\} .
\end{aligned}
$$

$A_{0}$ is defined after Eq. (24), $\mathrm{Li}_{4}[x]$ is the polylogarithm, and $\zeta[x]$ is the Riemann Zeta function.

Finally, there is one last three-loop CS equation, similar to (31), that involves $V^{(3, n)}$

$$
\begin{aligned}
\frac{\partial V^{(3, n)}}{\partial \ln z}+\beta_{\lambda_{j}}^{(1)} \frac{\partial V^{(2, n)}}{\partial \lambda_{j}}+\beta_{\lambda_{j}}^{(3, n)} \frac{\partial V}{\partial \lambda_{j}} & =\mathcal{O}\left(\lambda_{j}^{5}\right), \\
\lambda_{j} & =\lambda, \lambda_{6}, \lambda_{8},
\end{aligned}
$$

where $\beta_{\lambda}^{(3, n)}$ denotes possible three-loop corrections beyond $\beta_{\lambda}^{(3)}$. Equation (40) is actually a field-dependent condition. As usual $V^{(3, n)}$ only involves new field operators beyond $V^{(3)}$, suppressed by $\sigma$, e.g., $\phi^{6} / \sigma^{2}$, etc. The last term in the lhs with $\lambda_{j} \rightarrow \lambda$ would bring a term $\propto \phi^{4}$ which cannot be cancelled, being the only one of this structure. Then the only way to respect the above field-dependent condition is that $\beta_{\lambda}^{(3, n)}=0$. This is also seen from (40) in the decoupling limit of large $\langle\sigma\rangle$. Therefore, the three-loop beta function in the quantum scale invariant effective theory is just that of the theory with $\mu=$ constant. $^{18,19}$ We then integrate Eq. (40) using the replacement $\ln z \rightarrow(-1 / 2) \overline{\ln }\left(V_{\phi \phi} /(z \sigma)^{2}\right)$ which fixes the "constant" of integration in a scale invariant way. We find $^{20}$

$$
\begin{aligned}
V^{(3, n)}= & \frac{\lambda^{3}}{2 \kappa^{3}} \phi^{4}\left\{\left(27 \lambda-\frac{\lambda_{6}}{2}\right) \frac{\phi^{2}}{8 \sigma^{2}}+\left(\frac{401 \lambda}{72}-\lambda_{8}\right) \frac{\phi^{4}}{16 \sigma^{4}}\right\} \\
& \times \overline{\ln } \frac{V_{\phi \phi}}{(z \sigma)^{2}} .
\end{aligned}
$$

$V^{(3, n)}$ is correct up to a possible additional presence of a scale invariant $z$-independent three-loop finite (nonpolynomial) term $\left(\lambda^{4} / \kappa^{3}\right) \phi^{10} / \sigma^{6}$ that cannot be captured by the CS differential equation but only in the diagrammatic approach. In the limit of a large field $\sigma$ and similar to $V^{(2, n)}$ at two loop, $V^{(3, n)} \rightarrow 0$, leaving "usual" $V^{(3)}$ as the sole three-loop correction to the potential, with only a $\log$ dependence on $\sigma$.

To conclude, quantum scale invariance demands the presence of nonpolynomial operators. This symmetry

\footnotetext{
${ }^{18}$ Therefore, we have $\beta_{\lambda}^{(3)}=\lambda^{4} / \kappa^{3}(145 / 8+12 \zeta[3])$ [42-44].

${ }^{19}$ This is also consistent with $Z_{\sigma}=1$ at three loops. A threeloop wave function correction to $\sigma$ generated by a coupling $\epsilon \sigma \phi^{4}$ would then be proportional to $\propto \epsilon^{2} \times\left(1 / \epsilon^{2}\right)$, so no new poles emerge in this order.

${ }^{20}$ "Constants" of integration $\phi^{6} / \sigma^{2}, \phi^{8} / \sigma^{4}, \phi^{4}$ are not allowed, being "fixed" in (32), [(38), (39) for $\left.\phi^{4}\right]$.
}

arranges them in a series expansion in powers of $\phi / \sigma$ that contributes to the scalar potential. Each of these operators is actually an infinite sum of polynomial operators (in fields), after a Taylor expansion about $\sigma=\langle\sigma\rangle+\tilde{\sigma} . V^{(2, n)}, V^{(3, n)}$, $\Delta V$ are relevant for the behavior of the potential at large $\phi \sim \sigma$ and are suppressed at $\phi \ll \sigma$.

\section{E. More operators}

Having seen the scale invariant nonpolynomial operators generated at loop level, it is of interest to see their role if they are included in the action already at classical level, as in

$$
V=\frac{\lambda}{4 !} \phi^{4}+\frac{\lambda_{6}}{6} \frac{\phi^{6}}{\sigma^{2}}+\cdots
$$

where we ignore similar higher order terms. The last term breaks the enhanced Poincaré symmetry $\left(P_{v} \times P_{h}\right)$ only mildly, since this symmetry is restored at large $\sigma$. In a consistent setup like the Brans-Dicke-Jordan theory of gravity, this operator suppressed by $\langle\sigma\rangle \sim M_{\text {Planck }}$ could mediate gravitational interactions of the two sectors. Such an operator is also generated when going from the Jordan to Einstein frame, after a conformal transformation. ${ }^{21}$

The one-loop computation of the potential proceeds as before and has three contributions, all scale invariant. First, there is a one-loop contribution similar to that in Eq. (12) with $V_{\phi \phi}$ replaced by the (two) field-dependent (masses) ${ }^{2}$ which are eigenvalues of the matrix of second derivatives of $V$ above with respect to $\phi$ and $\sigma$, then sum over these.

A second contribution to the potential exists. The two field-dependent masses derived from $\tilde{V}$ of Eq. (4) with $V$ as above have a correction $\mathcal{O}(\epsilon)$ induced by $\lambda_{6}$; when this multiplies $1 / \epsilon$ of Eq. (9), it generates a finite correction $V^{(1, n)} \propto \lambda_{6}$ already at one loop

$$
V^{(1, n)}=\frac{\lambda_{6}}{6 \kappa} \phi^{4}\left(4 \lambda \frac{\phi^{4}}{\sigma^{4}}+24 \lambda_{6} \frac{\phi^{6}}{\sigma^{6}}+5 \lambda_{6} \frac{\phi^{8}}{\sigma^{8}}\right) .
$$

Finally, there are also one-loop counterterms, of the form $\left(Z_{\lambda_{p}}-1\right) \lambda_{p} \phi^{p} /\left(p \sigma^{p-4}\right)$, where $p=6,8,10,12$ and where $Z_{\lambda_{p}}=1+\gamma_{\lambda_{p}} /(\kappa \epsilon)$ and $\gamma_{\lambda_{6}}=9 \lambda, \gamma_{\lambda_{8}}=56 \lambda_{6} / \lambda_{8}, \gamma_{\lambda_{10}}=$ $20 \lambda_{6}^{2} / \lambda_{10}, \gamma_{\lambda_{12}}=3 \lambda_{6}^{2} / \lambda_{12}$. Therefore, the potential has a third contribution

$$
\Delta V=\sum_{p} \frac{\lambda_{p}}{p} \frac{\phi^{p}}{\sigma^{p-4}}, \quad p=6,8,10,12 .
$$

$V^{(1, n)}$ and $\Delta V$ are similar to $V^{(2, n)}, V^{(3, n)}, \Delta V$ found in the previous section, except that they are generated at one loop, due to nonzero $\lambda_{6}$. The one-loop beta functions of $\lambda_{p}$ are

$$
\beta_{\lambda_{p}}^{(1)}=\frac{2}{\kappa} \lambda_{p} \gamma_{\lambda_{p}}
$$

\footnotetext{
${ }^{21}$ We ignore here the effect of $\phi$ on the vev of $\sigma$.
} 
with $p$ as above, and they vanish if $\lambda_{6}=0$. We checked that the one-loop CS equation is again verified in the presence of these operators. For large $\langle\sigma\rangle$, dilaton fluctuations are suppressed and the above corrections to the potential vanish, to leave the "usual" result (first contribution above), obtained in the renormalizable theory with $\mu$ constant $(=z\langle\sigma\rangle)$. The generalization to more operators in the classical action is immediate.

\section{F. Symmetries, regularizations, and mass hierarchy}

From the above examples, we see that a combination of quantum scale invariance and enhanced Poincaré symmetry [39] of the two sectors can ensure a protection of the mass corrections to $\phi$ against a quadratic dependence on the scale of symmetry breaking $\langle\sigma\rangle$ (the only UV physical scale here). No term such as $\lambda \phi^{2} \sigma^{2}=\lambda\langle\sigma\rangle^{2} \phi^{2}+\cdots$ was generated at the quantum level in the potential, with $\lambda$ the Higgs self-coupling; if present, this would have required the usual SM-like fine-tuning of $\lambda$. Further, if one introduces a classical "mixing" coupling $\lambda_{m}$, with a tree-level term $\lambda_{m} \phi^{2} \sigma^{2}$ which would break the enhanced $P_{v} \times P_{h}$ symmetry, this would require a tuning of $\lambda_{m}$ (rather than $\lambda$ ) upon replacing $\sigma \rightarrow\langle\sigma\rangle+\tilde{\sigma}$, in order to keep the correction to the mass of $\phi$ under control. But such tuning is natural and needs to be done only once at the classical level, since the beta function $\beta_{\lambda_{m}} \sim \lambda_{m}$ at one loop [22,30,35,39] and two loops [36]. Further, for large $\langle\sigma\rangle$, the nonpolynomial operators that broke the $P_{v} \times P_{h}$ symmetry vanish, and this symmetry and its "protective" role are restored. Therefore, this protection remains true in the presence of nonpolynomial operators e.g., $\lambda_{6} \neq 0 .{ }^{22}$

The SR scheme used here is based on the DR scheme which may be considered unsuitable to capture the quadratic UV-scale dependence of the scalar (mass) ${ }^{2}$. It is important to note, however, that in our approach any scale is generated by fields vevs after spontaneous SSB. The field dependence (e.g., counterterms, etc.) of the quantum corrected action is not affected by the regularization and is actually dictated by the symmetries of the theory (including scale invariance), which our SR scheme respects (unlike DR). Therefore, the dependence of the quantum action on the mass scales (generated by these fields vev's) cannot be affected. The UV behavior of the mass of $\phi$, i.e., its dependence on $\langle\sigma\rangle$ (our physical UV scale) is thus not affected by a regularization that respected all symmetries of the theory. ${ }^{23}$

\footnotetext{
${ }^{22}$ Since we are using spontaneous SSB and a SR scheme, the conclusions of [47] do not apply here.

${ }^{23}$ To appreciate the role of $d=4$ enhanced Poincare symmetry, consider a different scale-invariant regularization that violates the $P_{v} \times P_{h}$ symmetry. For $V=\lambda \phi^{4} / 4$ !, use a momentum "cutoff" regularization: $k^{2} \leq \sigma^{2} ; \sigma$ is a hidden sector field with $\langle\sigma\rangle$ the scale of new physics. At one loop, $\Delta V \propto \int_{0}^{\sigma^{2}} d^{4} k \ln \left(1+\lambda \phi^{2} /\left(2 k^{2}\right)\right)=$ $\lambda \phi^{2} \sigma^{2}+\ldots$ This term (absent in our case) requires the "usual" order-by-order fine-tuning of a self-coupling $\lambda$.
}

\section{CONCLUSION}

Following the original idea of Englert et al. and using a perturbative approach, we examined the quantum implications of a regularization scheme that preserves the scale invariance of the classical theory. To this purpose, we demanded that the analytical continuation of the theory to $d=4-2 \epsilon$ preserves the scale symmetry of the $d=4$ action. This is possible under the additional presence of a dilaton field $(\sigma)$, the Goldstone mode of scale symmetry breaking. This field is classically decoupled from the visible sector, following an enhanced Poincaré symmetry of the two sectors, but there are nevertheless quantum effects.

The scale invariance in $d=4-2 \epsilon$ and the dilaton it demands have two main effects:

(a) introduce new "evanescent" interactions $(\propto \epsilon)$ which have quantum consequences;

(b) generate the subtraction scale $\mu \sim\langle\sigma\rangle$ after spontaneous scale symmetry breaking.

As a result, a scale invariant regularization is reformulated into an ordinary DR scheme of $\mu=$ constant $(\propto\langle\sigma\rangle)$ plus an additional field (dilaton) with an infinite series of evanescent couplings to the visible sector. When evanescent interactions multiply the poles of loop integrals, new quantum corrections (finite or infinite counterterms) are generated, not present in the quantum version of the same theory regularized with $\mu=$ constant (i.e., no dilaton, explicit breaking). These corrections, which also include the $\log$ terms in the potential (such as $\ln \sigma$ ) are scale invariant and have effects such as the transmission of scale symmetry breaking after its spontaneous breaking in the dilaton (hidden) sector or dilaton-dilaton scattering.

The scalar potential was computed at two loops by direct calculation and at three loops by integrating its CallanSymanzik equation. The result is scale invariant. It contains new loglike corrections (in the dilaton $\sigma$ ) similar to those obtained by naively replacing $\mu \rightarrow \sigma$ in the result obtained in the "usual" DR scheme with $\mu=$ constant. In addition, depending on the details of the classical theory, scale invariant nonpolynomial effective operators are also generated from one or two loops onwards, in a series of the form $\phi^{4} \times(\phi / \sigma)^{2 n}$. These operators are important for large field values $\phi \sim \sigma$ (and can be comparable to "standard" log terms of the loop corrections); the beta functions of their couplings were also computed.

These operators are a generic presence and can be understood via their Taylor series expansion about the scale $\langle\sigma\rangle \neq 0$ of spontaneous SSB, when they become polynomial. Scale symmetry acts at the quantum level as an organizing principle that resums the polynomial ones. Therefore, maintaining at the quantum level the scale symmetry of the classical action makes the theory nonrenormalizable. In the decoupling limit of the dilaton, these operators vanish, and one recovers the quantum result of a renormalizable theory with explicit SSB (if the classical theory was renormalizable). 
The role of the quantum scale symmetry and enhanced Poincaré symmetry in protecting a mass hierarchy $m_{\phi}^{2} \ll$ $\langle\sigma\rangle^{2}$ was reviewed. This protection cannot be affected by working in a regularization ultimately based on a DR scheme, because all scales and thus hierarchy thereof are generated by vev's of the fields present in the quantum corrected action (after spontaneous SSB); its counterterms, i.e., the fields dependence are dictated by the symmetries of the theory (including scale symmetry) that our regularization respects (unlike DR), hence the aforementioned protection. This remains true in the presence of the nonpolynomial terms (i.e., despite nonrenormalizability) since at large $\langle\sigma\rangle$ the enhanced Poincaré symmetry is restored. The study can be extended to gauge theories.

\section{ACKNOWLEDGMENTS}

We thank P. Olszewski for a discussion on the twoloop case.
[1] D. Ghilencea, H. M. Lee, and M. Park, Tuning supersymmetric models at LHC: A comparative analysis at two-loop level, J. High Energy Phys. 07 (2012) 046.

[2] D. Ghilencea and G. G. Ross, The fine-tuning cost of the likelihood in SUSY models, Nucl. Phys. B868, 65 (2013); D. M. Ghilencea, Fixing the EW scale in supersymmetric models after the Higgs discovery, Nucl. Phys. B876, 16 (2013).

[3] K. A. Meissner and H. Nicolai, Conformal symmetry and the standard model, Phys. Lett. B 648, 312 (2007).

[4] R. Foot, A. Kobakhidze, and R. R. Volkas, Electroweak Higgs as a pseudo-Goldstone boson of broken scale invariance, Phys. Lett. B 655, 156 (2007); R. Foot, A. Kobakhidze, K. L. McDonald, and R. R. Volkas, Neutrino mass in radiatively-broken scale-invariant models, Phys. Rev. D 76, 075014 ((2007).

[5] R. Foot, A. Kobakhidze, K. L. McDonald, and R. R. Volkas, A solution to the hierarchy problem from an almost decoupled hidden sector within a classically scale invariant theory, Phys. Rev. D 77, 035006 (2008).

[6] T. Hur, D. W. Jung, P. Ko, and J. Y. Lee, Electroweak symmetry breaking and cold dark matter from strongly interacting hidden sector, Phys. Lett. B 696, 262 (2011); S. Iso, N. Okada, and Y. Orikasa, Classically conformal $B-L$ extended standard model, Phys. Lett. B 676, 81 (2009); The minimal B-L model naturally realized at TeV scale, Phys. Rev. D 80, 115007 (2009); M. Holthausen, M. Lindner, and M. A. Schmidt, Radiative symmetry breaking of the minimal left-right symmetric model, Phys. Rev. D 82, 055002 (2010); P. Ko, Electroweak symmetry breaking and cold dark matter from hidden sector technicolor, Int. J. Mod. Phys. A 23, 3348 (2008).

[7] R. Foot, A. Kobakhidze, and R. R. Volkas, Stable mass hierarchies and dark matter from hidden sectors in the scaleinvariant standard model, Phys. Rev. D 82, 035005 (2010).

[8] L. Alexander-Nunneley and A. Pilaftsis, The minimal scale invariant extension of the standard model, J. High Energy Phys. 09 (2010) 021.

[9] T. Hur and P. Ko, Scale Invariant Extension of the Standard Model with Strongly Interacting Hidden Sector, Phys. Rev. Lett. 106, 141802 (2011).

[10] K. Ishiwata, Dark matter in classically scale-invariant two singlets standard model, Phys. Lett. B 710, 134 (2012).
[11] N. Okada and Y. Orikasa, Dark matter in the classically conformal B-L model, Phys. Rev. D 85, 115006 (2012).

[12] S. Iso and Y. Orikasa, TeV Scale B-L model with a flat Higgs potential at the Planck scale in view of the hierarchy problem, Prog. Theor. Exp. Phys. 2013, 023 B08 (2013).

[13] C. Englert, J. Jaeckel, V. V. Khoze, and M. Spannowsky, Emergence of the electroweak scale through the Higgs portal, J. High Energy Phys. 04 (2013) 060.

[14] M. Heikinheimo, A. Racioppi, M. Raidal, C. Spethmann, and K. Tuominen, Physical naturalness and dynamical breaking of classical scale invariance, Mod. Phys. Lett. A 29, 1450077 (2014).

[15] M. Heikinheimo, A. Racioppi, M. Raidal, C. Spethmann, and K. Tuominen, Dark supersymmetry, Nucl. Phys. B876, 201 (2013).

[16] T. Hambye and A. Strumia, Dynamical generation of the weak and dark matter scale, Phys. Rev. D 88, 055022 (2013).

[17] I. Bars, P. Steinhardt, and N. Turok, Local conformal symmetry in physics and cosmology, Phys. Rev. D 89, 043515 (2014).

[18] C. D. Carone and R. Ramos, Classical scale-invariance, the electroweak scale and vector dark matter, Phys. Rev. D 88, 055020 (2013).

[19] V. V. Khoze, Inflation and dark matter in the Higgs portal of classically scale invariant standard model, J. High Energy Phys. 11 (2013) 215.

[20] D. Gorbunov and A. Tokareva, Scale-invariance as the origin of dark radiation?, Phys. Lett. B 739, 50 (2014).

[21] E. Gabrielli, M. Heikinheimo, K. Kannike, A. Racioppi, M. Raidal, and C. Spethmann, Towards completing the standard model: Vacuum stability, electroweak symmetry breaking, and dark matter, Phys. Rev. D 89, 015017 (2014).

[22] K. Allison, C. T. Hill, and G. G. Ross, Ultra-weak sector, Higgs boson mass, and the dilaton, Phys. Lett. B 738, 191 (2014).

[23] A. Karam and K. Tamvakis, Dark matter and neutrino masses from a scale-invariant multi-Higgs portal, Phys. Rev. D 92, 075010 (2015).

[24] K. Allison, C. T. Hill, and G. G. Ross, An ultra-weak sector, the strong $C P$ problem and the pseudo-Goldstone dilaton, Nucl. Phys. B891, 613 (2015). 
[25] P. G. Ferreira, C. T. Hill, and G. G. Ross, Scale-independent inflation and hierarchy generation, Phys. Lett. B 763, 174 (2016).

[26] A. Karam and K. Tamvakis, Dark matter from a classically scale-invariant $S U(3)_{X}$, Phys. Rev. D 94, 055004 (2016).

[27] S. Weinberg, Gauge hierarchies, Phys. Lett. 82B, 387 (1979); G. 't Hooft, in Naturalness, Chiral Symmetry, and Spontaneous Chiral Symmetry Breaking, in Recent Developments in Gauge Theories: Proceedings, Cargesse, 1979, edited by G. 't Hooft et al., NATO Advanced Study Institutes Series (Plenum Press, New York, 1980), Vol. 59, p. 135.

[28] W. A. Bardeen, Reports No. FERMILAB-CONF-95-391-T, No. C95-08-27.3.

[29] F. Englert, C. Truffin, and R. Gastmans, Conformal invariance in quantum gravity, Nucl. Phys. B117, 407 (1976).

[30] M. Shaposhnikov and D. Zenhausern, Quantum scale invariance, cosmological constant and hierarchy problem, Phys. Lett. B 671, 162 (2009).

[31] M.E. Shaposhnikov and F. V. Tkachov, Quantum scaleinvariant models as effective field theories, arXiv: 0905.4857

[32] R. Armillis, A. Monin, and M. Shaposhnikov, Spontaneously broken conformal symmetry: Dealing with the trace anomaly, J. High Energy Phys. 10 (2013) 030.

[33] C. Tamarit, Running couplings with a vanishing scale anomaly, J. High Energy Phys. 12 (2013) 098; See also related [39] on naturalness in such theories.

[34] D. Ghilencea, Manifestly scale-invariant regularization and quantum effective operators, Phys. Rev. D 93, 105006 (2016); One-loop potential with scale invariance and effective operators, Proc. Sci., CORFU2015 (2016) 040.

[35] D. Ghilencea, Z. Lalak, and P. Olszewski, Standard model with spontaneously broken quantum scale invariance, Phys. Rev. D 96, 055034 (2017).

[36] D. Ghilencea, Z. Lalak, and P. Olszewski, Two-loop scaleinvariant scalar potential and quantum effective operators, Eur. Phys. J. C 76, 656 (2016).
[37] F. Gretsch and A. Monin, Perturbative conformal symmetry and dilaton, Phys. Rev. D 92, 045036 (2015).

[38] P. G. Ferreira, C. T. Hill, and G. G. Ross, Weyl current, scale-invariant inflation and Planck scale generation, Phys. Rev. D 95, 043507 (2017).

[39] R. Foot, A. Kobakhidze, K. L. McDonald, and R. R. Volkas, Poincaré protection for a natural electroweak scale, Phys. Rev. D 89, 115018 (2014).

[40] S. Fubini, A new approach to conformal invariant field theories, Nuovo Cimento Soc. Ital. Fis. A 34, 521 (1976).

[41] Z. Komargodski and A. Schwimmer, On renormalization group flows in four dimensions, J. High Energy Phys. 12 (2011) 099.

[42] R. Shrock, Study of the six-loop beta function of the $\lambda \phi^{4}$ theory, Phys. Rev. D 94, 125026 (2016), and references therein.

[43] M. V. Kompaniets and E. Panzer, Minimally subtracted six loop renormalization of $O(n)$-symmetric $\phi^{4}$ theory and critical exponents, Phys. Rev. D 96, 036016 (2017).

[44] H. Kleinert, J. Neu, V. Schulte-Frohlinde, K. G. Chetyrkin, and S. A. Larin, Five loop renormalization group functions of $\mathrm{O}(\mathrm{n})$ symmetric $\phi^{4}$ theory and epsilon expansions of critical exponents up to $\epsilon^{5}$, Phys. Lett. B 272, 39 (1991); Erratum, Phys. Lett. B 319, 545(E) (1993).

[45] See, for example, J. M. Chung and B. K. Chung, Three loop effective potential of $\mathrm{O}(\mathrm{N}) \phi^{4}$ theory, J. Korean Phys. Soc. 33, 643 (1998), and references therein; J. M. Chung and B. K. Chung, Three loop renormalization of the effective potential, Phys. Rev. D 56, 6508 (1997); Erratum, Phys. Rev. D 59, 109902(E) (1999); J. M. Chung and B. K. Chung, Renormalization group improvement of the effective potential in massive $\phi^{4}$ theory: Next-next-next-toleading logarithm resummation, J. Korean Phys. Soc. 39, 971 (2001).

[46] S. P. Martin, Effective potential at three loops, Phys. Rev. D 96, 096005 (2017).

[47] G. Marques Tavares, M. Schmaltz, and W. Skiba, Higgs mass naturalness and scale invariance in the UV, Phys. Rev. D 89, 015009 (2014). 